\title{
ANÁLISE MORFOLÓGICA DO APARELHO DE VENENO NOS BRACONIDAE CYCLOSTOME (HYMENOPTERA)
}

\author{
Sandra Maria Barbalho ${ }^{1}$ \\ Angélica Maria Penteado-Dias ${ }^{1}$
}

\begin{abstract}
Venom apparatus morphology of Cyclostome Braconidae (HYMENOPTERA). The venom apparatus morphology of Braconidae can be useful in systematic and phylogenetical studies. This work shows a morphological analysis of the venom apparatus of the following cyclostome Braconidae subfamilies: Alysiinae, Aphidiinae, Braconinae, Doryctinae, Opiinae and Rogadinae. The characters studied are muscle, form and constrictions of the reservoir, and also number, form and branching of the glands.

KEY WORDS. Hymenoptera, Braconidae, cyclostome, venom apparatus
\end{abstract}

Os Braconidae incluem mais de 40.000 espécies distribuídas pelo mundo, o que se aproxima do total de todas as espécies de vertebrados juntas (ACHTERBERG 1988). É um grupo monofilético que pode ser separado do seu grupo irmão, os Ichneumonidae, por pelo menos dois caracteres sinapomórficos: a rígida junção do segundo e terceiro tergitos do metassoma e a ausência da segunda nervura recorrente. Os Braconidae têm sido amplamente utilizados em programas de controle biológico de pragas em agroecossistemas tropicais e subtropicais, portanto precisam ser largamente estudados para que se possa utilizá-los de forma segura e eficaz.

Os Braconidae podem ser separados basicamente em dois grupos (SHARKEY 1993): os não cyclostome e os cyclostome. Os primeiros possuem o labro não côncavo e usualmente esculturado. São endoparasitóides e a maioria é coinobionte. Os cyclostome possuem o labro normalmente côncavo e a maioria constitui-se de ectoparasitóides idiobiontes de larvas de Lepidoptera e Coleoptera. As subfamílias estudadas neste trabalho pertencem a este segundo grupo.

Os parasitóides de um modo geral podem regular o estado fisiológico de seus hospedeiros usando para tal a injeção de veneno pelo ovipositor. As funções do aparelho de veneno dentro dos Hymenoptera, no entanto, é pouco conhecida. $\mathrm{Na}$ maioria dos Parasitica é utilizado para paralisar ou matar outros artrópodos que lhes servirão de hospedeiros (ROBERTSON 1968). A paralisação pode ser de curta duração (poucos minutos a uma hora) ou de longa duração (muitos dias a muitos meses) (PIEK \& THOMAS 1969). A paralisação do hospedeiro facilita a oviposição do parasitóide.

1) Departamento de Ecologia e Biologia Evolutiva, Universidade Federal de São Carlos. Rodovia Washington Luiz Km 235, Caixa Postal 676, 13605-905 São Carlos, São Paulo, Brasil. 
A análise morfológica do aparelho de veneno tem grande importância em estudos de filogenia e classificação do grupo, assim como nos estudos de evolução do aparelho de veneno nos Hymenoptera, a evolução da paralisação do hospedeiro, a função do aparelho naquelas fêmeas que não paralisam seus hospedeiros e a relação entre o tipo de aparelho e a biologia do grupo em questão. Muitos autores utilizaram a morfologia do aparelho de veneno em suas análises filogenéticas, tais como ACHTERBERG $(1984,1988)$, QUiCKE \& ACHTERBERG (1990), WHARTON et al. (1992) e outros.

O aparelho de veneno possui um reservatório de veneno onde esta substância é estocada, filamentos glandulares onde é produzida e um ducto de veneno até o ovipositor (Fig. 1).

Nos Braconidae existem dois tipos de aparelho de veneno, denominados tipo 1 e tipo 2 (EDSON \& VINSON 1979). O aparelho do tipo 1 possui um reservatório de veneno com parede espessa de músculos circulares e longitudinais bastante inervados e delineados por uma íntima cuticular relativamente grossa, como é observado dentro dos Braconidae cyclostome. O reservatório de veneno do aparelho do tipo 2 possui uma parede fina e com pouca musculatura, tal como se encontra nos Braconidae não cyclostome.

As fêmeas que apresentam aparelho do tipo 1 têm hábitos ectoparasitóides de larvas paralisadas. As possuidoras de aparelho do tipo 2 não paralisam seus hospedeiros. Neste caso não está bem definida a função do veneno. Talvez sua função afete o desenvolvimento fisiológico do hospedeiro (TANAKA 1987).

O objetivo deste trabalho é o de fazer uma análise morfológica do aparelho de veneno já que as subfamílias de Braconidae de um modo geral, apresentam diversas dificuldades relacionadas à identificação, tanto a nível específico e genérico como também a nível de subfamília. Este problema se agrava ainda mais pois a região Neotropical é muito rica e diversa e as chaves de identificação existentes não são facilmente aplicáveis para os exemplares de nossa fauna. As subfamílias estudadas foram:

- Alysiinae - endoparasitóides coinobiontes solitários de larvas de Diptera;

- Aphidiinae - endoparastóides solitários coinobiontes de ninfas e adultos de Aphidiidae;

- Braconinae - maioria é ectoparasitóide idiobionte de larvas de Coleoptera e Lepidoptera;

- Doryctinae - maioria se constitui de ectoparasitóides idiobiontes de larvas de Coleoptera;

- Opiinae - endoparastóides solitários de larvas de Diptera;

- Rogadinae - podem ser idiobiontes ou coinobiontes, endo ou ectoparasitóides de larvas de Coleoptera e Lepidoptera.

As duas outras subfamílias pertencentes à linhagem cyclostome (Apozyginae e Gnamptodontinae) não foram incluídas no estudo por não se possuir exemplares para análise. 

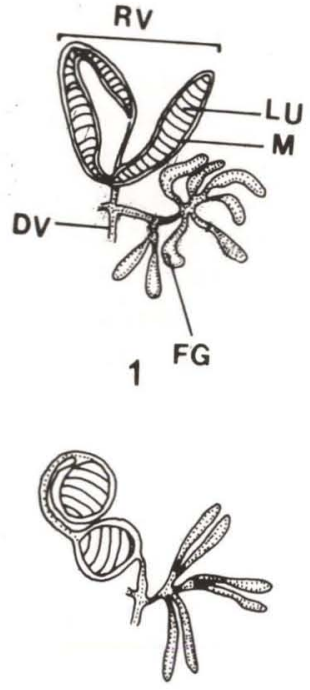

6

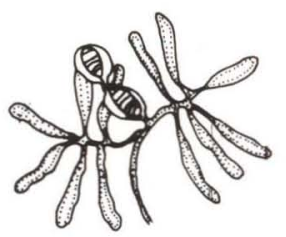

10

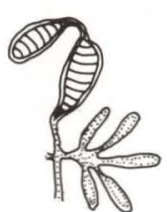

2

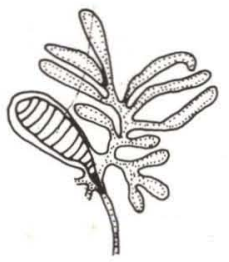

7

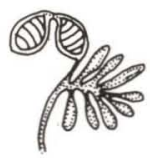

11

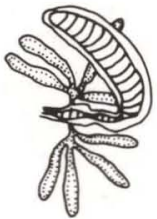

3

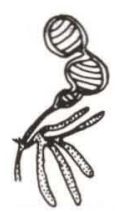

8

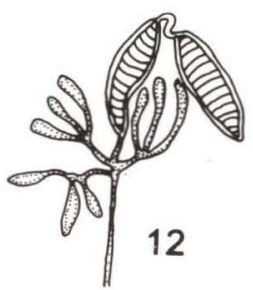

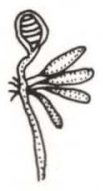

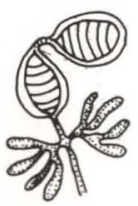

4

5

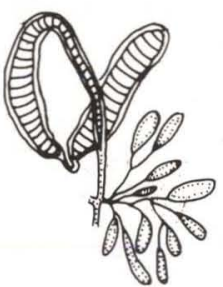

9

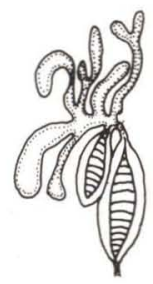

14

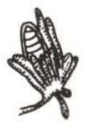

15

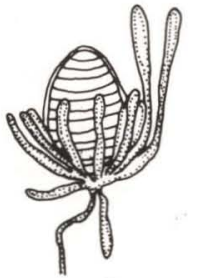

16

Figs 1-16. Aparelhos de veneno. (1-12) Doryctinae, onde (rv) reservatório de veneno, (lu) lúmen, (m) musculatura, (dv) ducto de veneno e (fg) filamento glandular. (1-2) Acrophasmus spp.; (3-7) Allorhogas spp.; (8-12) Heterospilus spp.; (13-16) Braconinae. (13) Digonogastra sp.; (14) Bracon sp.; (15) Ipobracon sp.; (16) Cyanopterus sp. 


\section{MATERIAL E MÉTODOS}

O material utilizado neste trabalho foi obtido a partir de coletas feitas com diferentes tipos de armadilhas em áreas de mata e cerrado da Fazenda Canchim, Empresa Brasileira de Pesquisa Agropecuária (EMBRAPA), São Carlos, São Paulo e mantido na coleção do Departamento de Ecologia e Biologia Evolutiva da Universidade Federal de São Carlos. As armadilhas utilizadas foram: Armadilha amarela de Água, Armadilha Malaise [modelo proposto por MALAISE (1937) e modificado por (TOWNES 1962)], Armadilha Suspensa (modelo de RAFAEL \& GORAYEB 1982) e "Varredura" da Vegetação. Este material conservado em álcool à $70 \%$ foi identificado com auxílio de chaves de identificação (ACHTERBERG 1990, 1993). Os exemplares foram dissecados com auxílio de estiletes e tesouras especiais sob microscópio estereoscópico e o aparelho de veneno foi desenhado com auxílio de câmara clara acoplada a um microscópio.

\section{RESULTADOS E DISCUSSÃO}

Os resultados apresentados abaixo mostram considerável diferença no aparelho de veneno das subfamílias estudadas. Concordam com a análise de EDSON \& VINSON (1979) que os Alysiinae, Aphidiinae, Braconinae, Doryctinae, Opiinae e Rogadinae possuem aparelho de veneno do tipo 1, no entanto, estes autores encontraram para os Aphidiinae também aparelho do tipo 2, situação não constatada nos gêneros estudados. A tabela I apresenta um resumo dos diferentes tipos de aparelhos de veneno nas subfamílias estudadas.

Tabela I. Características dos diferentes tipos de aparelhos de veneno encontrados nas subfamílias analisadas.

\begin{tabular}{|c|c|c|c|c|c|c|}
\hline & $\begin{array}{l}\text { Forma do } \\
\text { reservatório }\end{array}$ & $\begin{array}{l}\text { Constricçãa do } \\
\text { reservatório }\end{array}$ & $\begin{array}{l}\text { Inserçōes } \\
\text { filamentares }\end{array}$ & $\begin{array}{l}\text { Localização das } \\
\text { inserçōes } \\
\text { filamentares }\end{array}$ & $\begin{array}{l}\text { Forma dos } \\
\text { filamentos } \\
\text { glandulares }\end{array}$ & $\begin{array}{l}\text { Ramificação dos } \\
\text { filamentos } \\
\text { glandulares }\end{array}$ \\
\hline $\begin{array}{l}\text { Doryctinae } \\
\text { Figs } 1-12\end{array}$ & $\begin{array}{l}\text { Circular, oval ou } \\
\text { bastante } \\
\text { alongada }\end{array}$ & $\begin{array}{l}\text { Presente ou } \\
\text { ausente }\end{array}$ & $\begin{array}{l}\text { Únicas ou } \\
\text { múltiplas }\end{array}$ & $\begin{array}{l}\text { Ducto ou } \\
\text { basalmente no } \\
\text { reservatório }\end{array}$ & $\begin{array}{l}\text { Tubulosa ou } \\
\text { globulosa }\end{array}$ & $\begin{array}{l}\text { Muito ou pouco } \\
\text { ramificados }\end{array}$ \\
\hline $\begin{array}{l}\text { Braconidae } \\
\text { Figs } 13-16\end{array}$ & Oval & $\begin{array}{l}\text { Presente ou } \\
\text { ausente }\end{array}$ & $\begin{array}{l}\text { Únicas ou } \\
\text { múltiplas }\end{array}$ & $\begin{array}{l}\text { Basal ou } \\
\text { medianamente } \\
\text { no reservatório }\end{array}$ & Tubulosa & Muito ramificados \\
\hline $\begin{array}{l}\text { Alysiinae } \\
\text { Figs } 17-23\end{array}$ & Oval & Ausente & $\begin{array}{l}\text { Únicas ou } \\
\text { múltiplas }\end{array}$ & $\begin{array}{l}\text { Ducto ou basal, } \\
\text { apical ou } \\
\text { subapicalmente } \\
\text { no reservatório }\end{array}$ & $\begin{array}{l}\text { Tubulosa ou } \\
\text { globulosa }\end{array}$ & $\begin{array}{l}\text { Muito ou pouco } \\
\text { ramificados }\end{array}$ \\
\hline $\begin{array}{l}\text { Rogadinae } \\
\text { Figs } 24-30\end{array}$ & $\begin{array}{l}\text { Circular, oval ou } \\
\text { bastante } \\
\text { alongada }\end{array}$ & Ausente & $\begin{array}{l}\text { Únicas ou } \\
\text { múltiplas }\end{array}$ & $\begin{array}{l}\text { Ducto ou } \\
\text { basalmente no } \\
\text { reservatório }\end{array}$ & $\begin{array}{l}\text { Tubulosa ou } \\
\text { globulosa }\end{array}$ & $\begin{array}{l}\text { Ausente ou } \\
\text { muito ramificados }\end{array}$ \\
\hline $\begin{array}{l}\text { Aphidiinae } \\
\text { Fig. } 31\end{array}$ & Circular & Ausente & Múltiplas & Ducto & Tubulosa & Ausente \\
\hline $\begin{array}{l}\text { Opiinae } \\
\text { Figs } 32-34\end{array}$ & Oval & Ausente & $\begin{array}{l}\text { Únicas ou } \\
\text { múltiplas }\end{array}$ & $\begin{array}{l}\text { Ducto ou } \\
\text { basalmente no } \\
\text { reservatório }\end{array}$ & $\begin{array}{l}\text { Tubulosa ou } \\
\text { globulosa }\end{array}$ & $\begin{array}{l}\text { Muito ou pouco } \\
\text { ramificados }\end{array}$ \\
\hline
\end{tabular}

Os Doryctinae são os que englobam maior número de espécies estudadas e apresentaram as características descritas a seguir. $\mathrm{O}$ reservatório de veneno pode possuir (Figs 1-2, 5-6, 8-12) ou não (Figs 3-4, 7) constricções dividindo-o; pode ser 
de forma circular (Figs 4-6, 8, 10-11), oval (Figs 2, 7) ou bastante alongada (Figs $1,3,9,12$ ). Quanto às inserções filamentares, podem ser únicas (Fig. 11) ou múltiplas (Figs 1-10,12) e podem estar localizadas basalmente no reservatório de veneno (Figs 3, 7) ou no ducto de veneno (Figs 1-2, 4-6, 8-12). A forma dos filamentos glandulares é tubulosa (Figs 1-8, 10-12) ou elíptica (Fig. 9). Os filamentos podem ser muito (Figs 1-2, 6-11) ou pouco ramificados (Figs 3-5, 12), sendo considerados pouco ramificados os filamentos com até quatro ramos.

Os Braconinae apresentam reservatório de veneno oval que pode estar dividido (Fig. 14) ou não (Figs 13, 15, 16) por uma constricção. Os filamentos glandulares são tubulosos, muito ramificados (Figs 13-16) e sua inserção pode ser única (Fig. 14) ou múltipla (Figs 13, 15, 16). As inserções filamentares, podem ser basais (Figs 13, 15, 16) ou medianas (Fig. 14) no reservatório de veneno.

Os Alysiinae apresentam reservatório de veneno de forma oval e sem constricções (Figs 17-23). As inserções filamentares podem ser únicas (Figs 18, 22) ou múltiplas (Figs 17, 19-21, 23). Os filamentos glandulares, que podem estar inseridos no reservatório de veneno (Figs 18, 19, 21, 23) ou no ducto de veneno (Figs 17, 20, 22), podem se apresentar sem (Fig. 20), com poucas (Figs 19, 21, 23) ou com muitas ramificações (Figs 17, 18, 22). Ainda com relação aos filamentos glandulares, podem estar inseridos basalmente (Figs 18, 21), apicalmente (Fig. 19) ou subapicalmente (Fig. 23) no reservatório de veneno. A forma dos filamentos varia de globulosa (Figs 17-19, 21) a tubulosa (Figs 20, 22, 23).

Nos Rogadinae, o reservatório de veneno não apresenta constriç̧ões (Figs 24-30) e sua forma pode ser circular (Fig. 25), oval (Figs 24, 26-28, 30) ou bastante alongada (Fig. 29). As inserções filamentares podem ser únicas (Fig. 29) ou múltiplas (Figs 24-28,30). Os filamentos glandulares também podem ser muito ramificados (Figs 24-29) ou não apresentarem qualquer ramificação (Fig. 30). A inserção dos filamentos glandulares ocorre no ducto ou basalmente no reservatório de veneno. Com relação à forma dos filamentos glandulares, foram encontrados tanto globulosos (Figs 24-28, 30) quanto tubulosos (Fig. 29).

Os Aphidiinae analisados (Fig. 31) apresentam reservatório de veneno circular e sem constriç̧ões; filamentos glandulares tubulosos sem ramificações e com inserção múltipla no ducto de veneno.

A subfamília Opiinae apresenta reservatório de formato oval com (Figs 32-33) e sem (Fig. 34) constricções. Os filamentos glandulares podem ser tubulosos (Figs 32-33) ou globulosos (Fig. 34) e estão inseridos basalmente no reservatório de veneno (Fig. 32) ou no ducto de veneno (Figs 33-34). Podem ser muito ramificados (Figs 32, 34) ou não possuírem qualquer ramificação (Fig. 33). As inserções filamentares são únicas (Fig. 34) ou múltiplas (Figs 32-33).

\section{CONCLUSÕES}

A análise morfológica aqui apresentada fornece informações importantes para o estudo filogenético dos grupos, tanto a nível de subfamília quanto a nível genérico e específico.

A variação na morfologia do aparelho de veneno pode trazer respostas 
definitivas sobre os caminhos evolutivos seguidos pelos diferentes taxa e a proximidade ou não deles. Além disso, estes estudos devem ser ampliados e estendidos aos demais grupos de Hymenoptera para que se possa ter um conhecimento global desta ordem no que se refere aos hábitos parasitóides, verificando-se os padrões de especialização do reservatório de veneno do parasitóide ao hospedeiro.

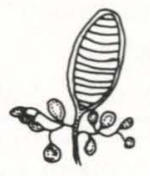

17

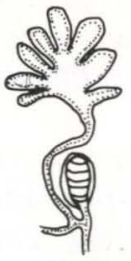

22

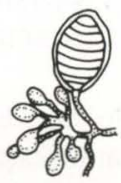

27

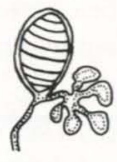

18

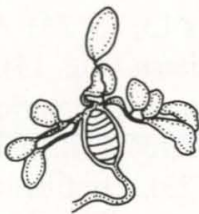

19

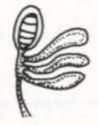

20

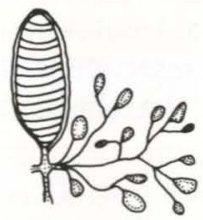

24

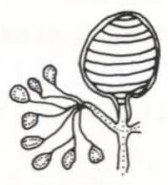

25

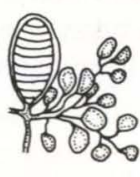

26

21
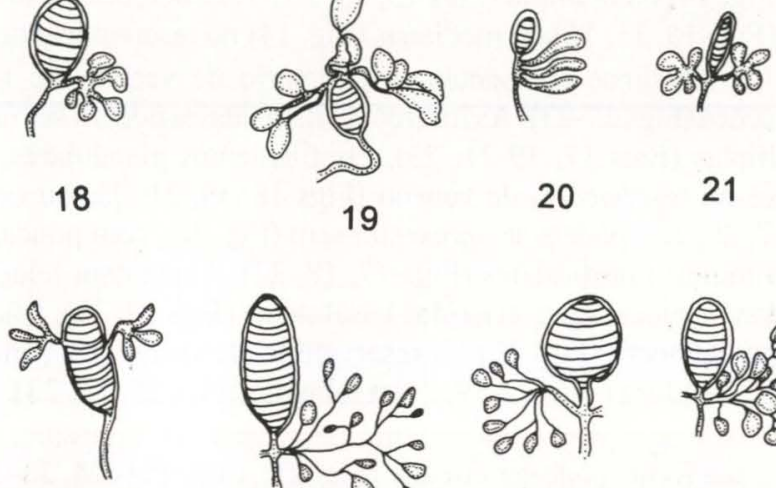

23

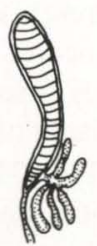

29

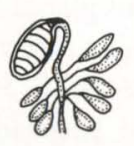

30

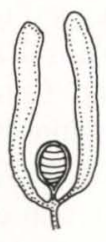

31

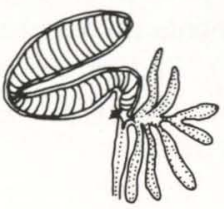

32

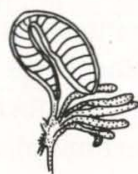

33

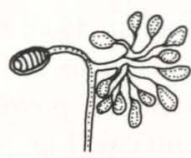

34

Figs 17-34. Aparelhos de veneno. (17-23) Alysiinae. (17-19) Aphaereta spp.; (20) Alysia sp.; (21) Idiasta sp.; (22-23) Aspilota spp. (24-30) Rogadinae. (24-28) Aleiodes spp.; (29-30) Rogas spp.; (31) Aphidiinae, Aphidius sp. (32-34) Opiinae. (32-33) Euopius spp.; (34) Opius sp. 


\section{REFERÊNCIAS BIBLIOGRÁFICAS}

ACHTERBERG, C. VAN. 1984. Essay on the Phylogeny of Braconidae (Hymenoptera, Ichneumonoidea). Tijdschr. Ent. 105: 41-48.

.1988. Parallelisms in the Braconidae (Hymenoptera) with special reference to the biology, p. 85-115. In: V.K. GUPTA (Ed.). Advances in parasitic Hymenoptera research.. Leiden, E. J. Brill.

-1990. Illustrated key to the subfamilies of Holarctic Braconidae (Hymenoptera: Ichneumonoidea). Zool. Med. Leiden 64: 1-20,

. 1993. Illustrated key to the subfamilies of the Braconidae (Hymenoptera, Ichneumonoidea). Zool. Verhandenlingen 283: 1-189.

EDSON, K.M. \& S.B. VINSON. 1979. A comparative morphology of the venom apparatus of female braconids (Hymenoptera: Braconidae). Can. Ent. 111: 1013-1024.

Malaise, R. 1969. A new insect-trap. Ent. Tidiscrif. 58: 148-160.

PIEK, T. \& R.T.S. Thomas. 1969. Paralyzing venoms of solitary wasps. Comp. Biochm. Physiol. 30: 13-31.

QUICKE, D.L.J. \& C. VAN ACHTERBERG. 1990. Phylogeny of the subfamilies of the family Braconidae (Hymenoptera, Icheumonoidea). Zool. Verh. Leiden 258: $1-95$.

RAFAEL, J.A. \& I.S. GORAYEB. 1982. Uma nova armadilha suspensa e primeiros registros de mutucas de copas de árvores. Acta Amazônica 12 (1): 232-235.

RoBERTSON, P.L. 1968. A morphological and functional study of the venom apparatus in representatives of some major groups of Hymenoptera. Aust. J. Zool. 16: 133-166.

SHARKEY, M.J. 1993. Family Braconidae, p.362-395. In: H. GOULET \& J.T. HUBER (Eds). Hymenoptera of the world: an identification guide to families. Otawa, Centre for Land and Biological Resorces Research, Research Branch Agriculture Canada Publication.

TANAKA, T. 1987. Calyx and venom fluids of Apanteles kariyai (Hymenoptera Braconidae) as factors which prolong the larval period of the host, Pseudaletia separata (Lepidoptera - Noctuidae). Ann. ent. Soc. Amer. 80: 530-533.

Townes, H. 1962. Design for a Malaise trap. Proc. Ent. Soc., London, 64 (4): 253-262.

Wharton, R.A.; S.R. SHAW; M.J. SharkeY; D.B. WAHL; J.B. WoOleY; J.B. WHITFIELD; P.M. MARSH \& J.W. JOHNSON. 1992. Phylogeny of the subfamilies of the family Braconidae (Hymenoptera, Ichneumonoidea): a reassessment. Cladistics 8: 199-235.

Recebido em 25.VII.1996; aceito em 13.II.1997. 